Series A

\author{
I. MATHEMATICA
}

420

\title{
ON BOUNDARY \\ DERIVATIVES IN CONFORMAL MAPPING \\ BY
}

S. E. WARSCHAWSKI

H E L S I N K I 1968

S U O M A L A I N N T I E D A K A T E M I A

doi:10.5186/aasfm. 1968.420 
Communicated 12 January 1968 by Olli Lehto and Lauri Mrrberg 


\section{ON BOUNDARY DERIVATIVES IN CONFORMAL MAPPING*}

\section{Introduction}

In the following we present an elementary and simplified approach to the study of the differentiability of a conformal map at a boundary point, which at the same time permits us to improve upon existing results. Let $\Omega$ denote a simply connected domain, $z_{0}$ an accessible boundary point of $\Omega$ and $f$ a function which maps the upper half plane conformally onto $\Omega$ such that $z_{0}$ correspond to the origin $\zeta=0$. Since the papers by J. Wolff [20] in 1926 and particularly by C. Carathéodory [2] in 1929 appeared, a great deal of interest has centered on obtaining criteria for the existence of the (finite and non-vanishing) »angular derivate» of $f$ at $\zeta=0$. Such criteria were given by Ahlfors [1], the writer [17], Van der Corput [13], Visser [16] and more recently, [4], [5], by J. Ferrand and J. Dufresnoy, and others. A survey of the earlier criteria (until 1948) is presented in [9], Chapter III, and of later ones (until 1955) in [6], Chapter VI.

The derivations of most of these criteria are based on extensions of the Wolff-Carathéodory theorem in conjunction with one or both of Ahlfors' principal inequalities in [1] or on extensions and refinements of these inequalities (cf. [6], Chapter VI). The present writer has also been interested in [17], [18], and [19] in criteria for the existence of the "unrestricted》" derivative of $f$ at $\zeta=0$, assuming that the boundary of $\Omega$ in a neighborhood of $z_{0}$ is a (free) Jordan arc. Another proof of the author's result on the unrestricted derivative was given by M. Tsuji [12] pp. 366-377. A further method for providing the transition from the angular derivative to the unrestricted derivative is Ostrowski's »Faltensatz» [11]; see also the writer's recent new proof of this theorem [19]; cf. also Wolff's earlier result in $[21]$.

In the present paper we consider the inverse function $\phi$ of $f$ and show that under proper conditions (which in general cannot be improved) $\varphi$ has a finite derivative $x$ at $z=z_{0}$ for approach in the Ostrowski »kernel» of $\Omega$ at $z_{0}$ (for the definition of the kernel see section 1). This is the content of Theorem 1 (and it, of course, also implies the existence of the angular

*) Sponsored (in part) by the Office of Naval Research under contract Nom. 2216(28) with the University of California. 
derivative at $z_{0}$ ). However, here $\varkappa$ may be 0 and we give a rather general criterion (in section 5) which insures that $x \neq 0$.

From Theorem 1 we obtain in a very short manner a criterion for the existence of the unrestricted derivative (Theorem 2).

The method applied here to prove Theorem 1 is different from those previously employed in establishing criteria for the existence of the derivative, and the author believes it of interest even beyond the immediate purpose it serves in this paper.

\section{Preliminaries}

Let $\Omega$ be a simply connected domain in the $z$-plane, $z=x+i y$, and $z_{0}$ a boundary point at $z=0$ which is accessible along the segment $\left\{x=0,0<y \leqq r_{0}\right\} \subset \Omega$. For $0<r \leqq r_{0}$ let $k_{r}$ denote the subarc of $|z|=r$ which contains $z=i r$ and forms a (circular) cross cut of $\Omega$. Following A. Ostrowski [11] we denote the subdomain $K=\left\{z z \in k_{r}\right.$, $\left.0<r<r_{0}\right\}$ as the kernel of $\Omega$ at $z_{0}$. ( $K$ depends on $r_{0}$, but this is not essential for our purpose).

$\Omega$ will be, at times, subjected to the following additional conditions at $z_{0}$, although not necessarily simultaneously to all three.

(A) The boundary point $z_{0}$ is accessible in any "Stolz angle», symmetrical to the imaginary axis of opening less than $\pi$, i.e. for every $1,0<1<\frac{\pi}{2}$. there exists an $r_{\alpha}>0$ such that the sector $A_{\alpha}=\{z \gamma \leqq \arg z \leqq \pi-\lambda$, $\left.0<|z| \leqq r_{\alpha}\right\} \subset \Omega$.

If the length of $k_{r}$ is $r \Theta(r)$, and $\pi \varepsilon(r)=\operatorname{Max}[\Theta(r)-\pi .0]$. then the two other conditions are:

$$
\text { (B) } \quad \int_{0}^{r_{0}} \frac{\varepsilon(r)}{r} d r<\infty
$$

and

$$
\text { (C) } \quad \lim _{r \rightarrow 0} \varepsilon(r)=0 .
$$

Let $\zeta=\varphi(z), \quad \zeta=\xi+i \eta, \operatorname{map} \Omega$ conformally onto the half-plane $\eta>0$ such that $z_{0}$ corresponds to $\zeta=0$. We may assume that the endpoints of the image $\varphi\left(k_{r_{0}}\right)$ of $k_{r_{0}}$ are finite and on opposite sides of $\zeta=0$.

If $\Omega$ satisfies the hypotheses $(\mathrm{A})$ and $(\mathrm{C})$, then $\varphi(z)$ is ssemi-conformal» in the kernel $K$, i.e. as $z \rightarrow z_{0}$, $z \in K$

$$
\lim _{z \rightarrow z_{0}} \arg \frac{\psi(z)}{z}=0 \text {. }
$$


This is a direct consequence of a lemma of $\mathrm{J}$. Wolff [22], as stated (with proof) in [19], pp. 84-86. ${ }^{1}$ ) In [19] it is proved as a »Remark» to Theorem 1 a, p. 89; a direct proof is given in the Appendix of this paper.

\section{Auxiliary results}

We prove first the following lemmas.

Lemma 1. Suppose the domain $\Omega$ satisfies condition (B). Then there exists a constant $M$ such that for $\left.z \in K,|z| \leqq \frac{r_{0}}{2},\left|\frac{\varphi(z)}{z}\right| \leqq M .^{2}\right)$

Proof. Let $l_{\varrho}$ denote the length of the image $\gamma_{\varrho}=\varphi\left(k_{\varrho}\right)$ under the mapping $z \rightarrow \varphi(z)$. Then we have

$$
l_{\varrho}^{2}=\left(\int_{k_{\varrho}}\left|\varphi^{\prime}\left(\varrho e^{i \theta}\right)\right| \varrho d \theta\right)^{2} \leqq \int_{k_{\varrho}}\left|\varphi^{\prime}\left(\varrho e^{i \theta}\right)\right|^{2} \varrho d \theta \cdot \Theta(\varrho) \varrho .
$$

Hence, for $0<r \leqq r_{0}$, since $\Theta(\varrho) \leqq \pi(1+\varepsilon(\varrho))$,

$$
\int_{0}^{r} \frac{l_{\varrho}^{2} d \varrho}{\varrho(1+\varepsilon(\varrho))} \leqq \pi \int_{0}^{r} d \varrho \int_{k_{\varrho}}\left|\varphi^{\prime}\left(\varrho e^{i \theta}\right)\right|^{2} \varrho d \theta=\pi A(r) .
$$

Here $A(r)$ denotes the area of image $\Delta_{r}$ of $D_{r}$ and $D_{r}$ is that of the two subdomains of $\Omega$ formed by $k_{r}$ which contains the segment $0<y<r$ of the imaginary axis. For almost all $r, 0<r \leq r_{0}, l_{r}<\infty$ and $\gamma_{r}$ is therefore a rectifiable Jordan arc; $\Delta_{r}$ is bounded by $\gamma_{r}$ and a segment of the real axis (containing $\zeta=0$ ). We reflect $\gamma_{r}$ and $\Delta_{r}$ in the real axis, and $\gamma_{r}$ and its reflection $\bar{\gamma}_{r}$ form a closed Jordan curve of length $2 l_{r}$. The area of its interior is $2 A(r)$. Hence, we have by the isoperimetric inequality

$$
2 A(r) \leqq \frac{\left(2 l_{r}\right)^{2}}{4 \pi} \text { or } \pi A(r) \leqq \frac{l_{r}^{2}}{2}
$$

Thus we have for all $r, 0<r \leq r_{0},\left(l_{r}\right.$ may be $+\infty$ on a set of measure 0$)$

1) If $\Omega$ satisfies (A) and (B), then it is shown in [19], p. 96 that (1.1) holds as $z \rightarrow z_{0}$ in any angle $A_{\alpha}$. A necessary and sufficient condition that the map $z \rightarrow \varphi(z)$ be "semi-conformal" in any Stolz angle $A_{\alpha}$ is due to Ostrowski [11]; several proofs of this result were subsequently given by C. Gattegno [7], based on Wolff's lemma, in [22], J. Lelong Ferrand [6], pp. 113-122, by use of Carathéodory's »kernel» theorem, and by the writer [19], pp. 87-89.

2) The proof of this lemma may be obtained by use of Ahlfors' First Inequality (Distortion Theorem) [1]. However, we present an independent proof since some of the inequalities we obtain are used later on in this paper. 


$$
\pi \int_{0}^{r} \int_{k_{r}}\left|\varphi^{\prime}\left(\varrho e^{i \theta}\right)\right|^{2} \varrho d \theta d \varrho \leqq \frac{l_{r}^{2}}{2} .
$$

Since for almost all $r, 0 \leqq r \leqq r_{0}$

$$
A^{\prime}(r)=\int_{k_{r}}\left|\varphi^{\prime}\left(r e^{i \vartheta}\right)\right|^{2} r d \theta
$$

we have from $(2.1)$

$$
l_{r}^{2} \leqq \pi r(1+\varepsilon(r)) A^{\prime}(r)
$$

and by use of (2.3)

$$
A(r) \leqq \frac{r}{2}(1+\varepsilon(r)) A^{\prime}(r), \frac{2}{r(1+\varepsilon(r))} \leqq \frac{A^{\prime}(r)}{A(r)},
$$

or, finally, noting that $1-\varepsilon(r) \leqq(1+\varepsilon(r))^{-1}$,

$$
\frac{2}{r}-\frac{2 \varepsilon(r)}{r} \leqq \frac{A^{\prime}(r)}{A(r)} \text {. }
$$

Integration between the limits $r$ and $r_{1}, r<r_{1} \leqq r_{0}$, yields

$$
\frac{A(r)}{r^{2}} e^{2 \int_{0}^{r} \frac{\varepsilon(t)}{t} d t} \leqq \frac{A\left(r_{1}\right)}{r_{1}^{2}} e^{2 \int_{0}^{r_{1}} \frac{\varepsilon(t)}{t} d t} .
$$

Because of condition (B) and the monotone character of the function above,

$$
\lim _{r \downarrow 0} \frac{A(r)}{r^{2}}=\mu \quad(\geqq 0)
$$

exists.

Now by (2.2), since clearly $0 \leqq \varepsilon(r) \leqq 1, \quad 0<r \leqq r_{0}$,

$$
\frac{1}{2} \int_{\frac{r}{2}}^{r} \frac{l_{t}^{2}}{t} d t<\int_{0}^{r} \frac{l_{t}^{2} d t}{t(1+\varepsilon(t))} \leqq \pi A(r) \leqq M_{0} r^{2}
$$

where $M_{0}=\pi \frac{A\left(r_{0}\right)}{r_{0}^{2}} \exp \left[2 \int_{0}^{r_{0}} \frac{\varepsilon(t)}{t} d t\right]$. Hence there exists an $r_{1}$ with $\frac{r}{2} \leqq r_{1} \leqq r$ such that

$$
i_{r_{1}}^{2} \log 2 \leqq 2 M_{0} r^{2}\left(\frac{r}{2} \leqq r_{1} \leqq r\right)
$$


Consider a cross cut $k_{\varrho}$ with $0<\varrho \leqq \frac{r_{0}}{2}$. We take $r=2 \varrho$ and apply (2.7), where now $\varrho \leqq r_{1} \leqq 2 \varrho$. Since $k_{\varrho}$ is contained in the closure of $D_{r_{1}}$ it follows that, for $z \in k_{0}, \varphi(z) \in A_{r_{1}}$ and therefore $\varphi(z) \leqq l_{r_{1}}$. Hence

$$
\left|\frac{\varphi(z)}{z}\right| \leqq \frac{l_{r_{1}}}{\varrho} \leqq 2 \sqrt{\frac{2 M_{0}}{\log 2}} \equiv M \quad\left(z \in k_{\varrho}\right)
$$

Lemma 2.3) If the mapping function $\varphi$ in Section 1 is "semiconformal» in any Stolz angle $A_{\alpha}\left(0<\alpha<\frac{\pi}{2}\right)$, i.e. if $\lim _{z \rightarrow z_{0}} \arg \frac{\varphi(z)}{z}=0$ in any $A_{\alpha}$,
then

$$
\lim _{z \rightarrow z_{0}} \frac{z \varphi^{\prime}(z)}{\varphi(z)}=1, z \in A_{\alpha}
$$

Furthermore if $\Omega$ satisfies conditions (A) and (B), then for $z \in A_{x}$

$$
\lim _{z \rightarrow z_{0}}\left[\frac{\varphi(z)}{z}-\varphi^{\prime}(z)\right]=0 \quad\left(z \in A_{\alpha}\right)
$$

Proof. Although (2.8) is well known ${ }^{3}$ ) we present a very short proof here for the sake of completeness. Let $\log \frac{z \varphi^{\prime}(z)}{\varphi(z)}$ represent the determination of the logarithm for which $\lim _{z \rightarrow z_{0}} \arg \frac{\varphi(z)}{z}=0 \quad\left(z \in A_{\alpha}\right)$. Let $z \in A_{x}$ and let $\varrho$ denote the radius of the largest circle about $z$ contained in $A_{x 2}$. Then (see e.g. [3] p. 88)

$$
i\left[\frac{\varphi^{\prime}(z)}{\varphi(z)}-\frac{1}{z}\right]=-\frac{1}{\pi \varrho} \int_{0}^{2 \cdot \tau} \arg \left[\frac{\varphi(\zeta)}{\zeta}\right] e^{-i \theta} d \theta \quad\left(\zeta=z+g e^{i \rho}\right)
$$

If $z$ is sufficiently close to $0, \frac{\varrho}{|z|} \geqq \sin \frac{\alpha}{2}$ and hence

$$
\left|\frac{z \varphi^{\prime}(z)}{\varphi(z)}-1\right| \leqq 2\left|\frac{\mathrm{z}}{\varrho}\right| \cdot h \leqq 2 h \csc \frac{x}{2}, h=\operatorname{Max} \arg \frac{\varphi(\zeta)}{\zeta}
$$

for $\zeta \in A_{\alpha / 2}$, and $|\zeta| \leqq|z|+\varrho$. As $z \rightarrow 0$ in $A_{\alpha}, \quad h \rightarrow 0$.

The second part of the lemma, (2.9), follows from the first by use of Lemma 1, since

3) C. Visser [14], theorem 7; A. Ostrowski [10], theorem 4. 


$$
\left|\varphi^{\prime}(z)-\frac{\varphi(z)}{z}\right|=\left|\left(\frac{z \varphi^{\prime}(z)}{\varphi(z)}-1\right) \frac{\varphi(z)}{z}\right| \leqq M\left|\frac{z \varphi^{\prime}(z)}{\varphi(z)}-1\right| .
$$

Lemma 3. Suppose $\Omega$ satisfies conditions (A), (B) and (C). Then there exist for every $r \leqq \frac{r_{0}}{2}$ and for every fixed $\delta, 0<\delta<1$ an $r^{\prime}$ with $r \leqq r^{\prime} \leqq r(1+\delta)$ and an $r^{\prime \prime}$ with $(1-\delta) r \leqq r^{\prime \prime} \leqq r$ such that for $\varrho=r^{\prime}$ and $\varrho=r^{\prime \prime}$

$$
\sigma(\varrho)=\int_{k_{\varrho}}\left|\varphi^{\prime}(z)-\frac{\varphi(z)}{z}\right| d \theta \rightarrow 0 \text { as } r \rightarrow 0 \quad\left(z=\varrho e^{i \theta}\right) .
$$

Corollary. For $z_{1} \in k_{\varrho}, z_{2} \in k_{\varrho}$, for $\varrho=r^{\prime}$ and $\varrho=r^{\prime \prime}$ uniformly:

$$
\lim _{r \rightarrow 0}\left|\frac{\varphi\left(z_{2}\right)}{z_{2}}-\frac{\varphi\left(z_{1}\right)}{z_{1}}\right|=0
$$

The Corollary follows immediately from the Lemma, since

$$
\left|\frac{\varphi\left(z_{2}\right)}{z_{2}}-\frac{\varphi\left(z_{1}\right)}{z_{1}}\right|=\left|\int_{\theta_{1}}^{\theta_{2}} \frac{\partial}{\partial \theta}\left[\frac{\varphi\left(\varrho e^{i \theta}\right)}{\varrho e^{i \theta}}\right] d \theta\right| \leqq \int_{k_{g}}\left|\varphi^{\prime}(z)-\frac{\varphi(z)}{z}\right| d \theta .
$$

Proof of the Lemma. Let $r e^{i \beta_{1}}$ and $r e^{i \beta_{2}}$ be the endpoints of $k_{r}$ and let $A_{\alpha}$ be a sector intersected by $k_{r}\left(0<\alpha<\frac{\pi}{2}\right)$. Then

$$
\sigma(r)=\int_{\beta_{1}}^{\beta_{2}}=\int_{\beta_{1}}^{\alpha}+\int_{\alpha}^{\pi-\alpha}+\int_{\pi-\alpha}^{\beta_{2}}
$$

Let $\eta>0$ be given. Then by Lemma 2 , for fixed $\alpha$,

$$
\int_{\alpha}^{\pi-\alpha}\left|\varphi^{\prime}(z)-\frac{\varphi(z)}{z}\right| d \theta<\eta \quad\left(z=r e^{i \theta}\right)
$$

for all sufficiently small $r$, say $r \leqq r_{1}(\eta, \alpha)$. Next, by Lemma 1 and the Schwarz inequality (for $z=r e^{i \theta}$ )

$$
\begin{aligned}
\int_{\beta_{1}}^{\alpha}\left|\varphi^{\prime}(z)-\frac{\varphi(z)}{z}\right| d \theta & \leqq \int_{\beta_{1}}^{\alpha}\left|\frac{\varphi(z)}{z}\right| d \theta+\int_{\beta_{1}}^{\alpha}\left|\varphi^{\prime}(z)\right| d \theta \\
& \leqq\left(\alpha-\beta_{1}\right) M+\left\{\left(\alpha-\beta_{1}\right) \int_{\beta_{1}}^{\alpha}\left|\varphi^{\prime}(z)\right|^{2} d \theta\right\}^{1 / 2}
\end{aligned}
$$


and an analogous estimate is obtained for $\int_{-\alpha}^{\beta_{2}}\left|\varphi^{\prime}(z)-\frac{\varphi(z)}{z}\right| d \theta$.

Noting that

$$
\alpha-\beta_{1} \leqq \alpha+\pi \varepsilon(r), \quad \beta_{2}-(\pi-\alpha) \leqq \alpha+\pi \varepsilon(r)
$$

we finally obtain

$$
\sigma(r) \leqq \eta+2(\alpha+\pi \varepsilon(r)) M+2\left\{(\alpha+\pi \varepsilon(r)) \int_{k_{r}} \mid \varphi^{\prime}\left(r e^{i \theta}\right)^{2} d \theta\right\}^{1 / 2}
$$

Now we determine a bound for the last integral. Since for $r \leqq r_{0}$,

$$
\frac{A(r)}{r^{2}}<\frac{A\left(r_{0}\right)}{r_{0}^{2}} \exp \left[2 \int_{0}^{r_{0}} \frac{\varepsilon(t)}{t} d t\right] \equiv M_{1}
$$

we have for $r \leqq \frac{r_{0}}{2}$

$$
\frac{A(r(1+\delta))-A(r)}{r^{2}}=\frac{A(r(1+\delta))}{r^{2}(1+\delta)^{2}}(1+\delta)^{2}-\frac{A(r)}{r^{2}}<4 M_{1}
$$

or

$$
\frac{1}{r^{2}} \int_{r}^{\mathrm{r}(1+\delta)} \int_{k_{t}}\left|\varphi^{\prime}\left(t e^{i \theta}\right)\right|^{2} t d \theta d t<4 M_{1}
$$

Hence, there exists an $r^{\prime}$ with $r \leqq r^{\prime} \leqq r(1+\delta)$ such that

$$
\int_{k_{\varrho}}\left|\varphi^{\prime}\left(\varrho e^{i \theta}\right)\right|^{2} d \theta \frac{1}{r^{2}} \int_{r}^{r(1+\delta)} t d t<4 M_{1} \quad\left(\varrho=r^{\prime}\right)
$$

or

$$
\int_{k_{\varrho}} \mid \varphi^{\prime}\left(\left.\varrho e^{i \theta}\right|^{2} d \theta \leqq \frac{8 M_{1}}{2 \delta+\delta^{2}}=K(\delta) \quad\left(\varrho=r^{\prime}\right) .\right.
$$

Thus, substituting $\varrho$ in place of $r$ in (2.11) and using (2.13) we obtain

$$
\varlimsup_{r \rightarrow 0} \sigma(\varrho) \leqq \eta+2 \alpha M+2\{\alpha K(\delta)\}^{1 / 2} \quad\left(\varrho=r^{\prime}\right) .
$$

Letting here first $\eta \rightarrow 0$ and subsequently (for fixed $\delta$ ) $\alpha \rightarrow 0$ we obtain 
the conclusion for $\varrho=r^{\prime}$. The derivation of a bound for the integral in (2.13) for $\varrho=r^{\prime \prime}$ and the remainder of the proof is completely analogous.

Lemma 4. Suppose the domain $\Omega$ and $k_{r}, \Theta(r), \varphi(z)$ are defined as in section 1. Let $l_{\boldsymbol{r}}$ be the length of $\varphi\left(k_{r}\right)$. Then for $0<r<r_{\mathbf{0}}$

$$
r \Theta(r) \operatorname{Inf}_{z \in k_{\boldsymbol{r}}}\left|\frac{\varphi(z)}{z}\right|-r \sigma(r) \leqq l_{r} \leqq r \Theta(r) \operatorname{Sup}_{z \in k_{r}}\left|\frac{\varphi(z)}{z}\right|+r \sigma(r) .
$$

Here $l_{r}$ and $\sigma(r)$ may be $+\infty$ for some $r$ on a set of measure 0 .

Proof. Consider a partition $\theta_{0}<\theta_{1}<\ldots<\theta_{n}$ of $k_{r}, z_{v}=r e^{i \theta_{v}}$. Then

$$
\begin{aligned}
L_{n} & =\sum_{v=0}^{n-1}\left|\varphi\left(z_{v+1}\right)-\varphi\left(z_{v}\right)\right|=\sum_{v=0}^{n-1}\left|\frac{\varphi\left(z_{v+1}\right)}{z_{v+1}} z_{v+1}-\frac{\varphi\left(z_{v}\right)}{z_{v}} z_{v}\right| \\
& \leqq \sum_{v=0}^{n-1}\left|\frac{\varphi\left(z_{v+1}\right)}{z_{v+1}}-\frac{\varphi\left(z_{v}\right)}{z_{v}}\right|\left|z_{v+1}\right|+\sum_{v=0}^{n-1}\left|\frac{\varphi\left(z_{v}\right)}{z_{v}}\right|\left|z_{v+1}-z_{v}\right|
\end{aligned}
$$

Hence

$$
L_{n} \leqq \operatorname{Sup}_{z \in k_{r}}\left|\frac{\varphi(z)}{z}\right| \cdot r \Theta(r)+r \sum_{\nu=0}^{n-1} \int_{\theta_{\nu}}^{\theta_{v+1}}\left|\varphi^{\prime}\left(r e^{i \theta}\right)-\frac{\varphi\left(r e^{i \theta}\right)}{r e^{i \theta}}\right| d \theta
$$

or

$$
l_{r} \leqq r \Theta(r) \operatorname{Sup}_{z \in k_{r}}\left|\frac{\varphi(z)}{z}\right|+r \sigma(r) .
$$

Since also

$$
L_{n} \geqq \sum_{\nu=0}^{n-1}\left|\frac{\varphi\left(z_{v}\right)}{z_{v}}\right|\left|z_{v+1}-z_{v}\right|-r \sigma(r)
$$

we find easily, by letting the norm of the partition tend to 0 , the left hand inequality.

\section{The derivative for approach in the kernel}

We prove now our first principal theorem.

Theorem 1. If the domain $\Omega$ satisfies hypotheses (A), (B), and (C) and if $q(z)$ is the mapping function defined in section 1 , then for $z \in K$,

$$
\lim _{z \rightarrow z_{0}} \frac{\varphi(z)}{z}=x=\sqrt{\frac{2 \mu}{\pi}} .
$$

Here $\mu=\lim _{r \rightarrow 0} \frac{A(r)}{r^{2}}$ defined in (2.5). 
Proof. For any $r, 0<r \leqq \frac{r_{0}}{2}$, and fixed $\delta, 0<\delta<1$, let $\varrho=r^{\prime}$ or $\varrho=r^{\prime \prime}$ (Lemma 3). Then by $(2.10)$

$$
\operatorname{Sup}_{z \in k_{\varrho}}\left|\frac{\varphi(z)}{z}-\frac{\varphi(i \varrho)}{i \varrho}\right|=\eta=\eta(\varrho, \delta) \rightarrow 0 \text { as } \varrho \rightarrow 0 .
$$

Hence, by (2.14)

$$
\left(\left|\frac{\varphi(i \varrho)}{i \varrho}\right|-\eta\right) \Theta(\varrho)-\sigma(\varrho) \leqq \frac{l_{\varrho}}{\varrho} \leqq\left(\left|\frac{\varphi(i \varrho)}{i \varrho}\right|+\eta\right) \Theta(\varrho)+\sigma(\varrho) .
$$

As $r \rightarrow 0$ we have, therefore, for $\varrho=r^{\prime}$ and $\varrho=r^{\prime \prime}$ :

$$
\varlimsup_{r \rightarrow 0}\left|\frac{\varphi(i \varrho)}{i \varrho}\right| \pi \leqq \varlimsup_{r \rightarrow 0} \frac{l_{\varrho}}{\varrho}, \quad \frac{\lim }{r \rightarrow 0}\left|\frac{\varphi(i \varrho)}{i \varrho}\right| \pi \geqq \frac{\lim }{r \rightarrow 0} \frac{l_{\varrho}}{\varrho} .
$$

Now, by (2.3), for $0<r<r_{0}$

$$
2 \pi \frac{A(r)}{r^{2}} \leqq \frac{l_{r}^{2}}{r^{2}}
$$

and, therefore, by (2.5)

$$
2 \pi \mu \leqq \frac{\lim }{r \rightarrow 0} \frac{l_{r}^{2}}{r^{2}} .
$$

Thus, by the second inequality in (3.3)

$$
\sqrt{\frac{2 \mu}{\pi}} \leqq \frac{\lim }{\varrho \rightarrow 0}\left|\frac{\varphi(i \varrho)}{i \varrho}\right|
$$

To estimate the $\varlimsup_{\varrho \rightarrow 0} \frac{l_{\varrho}}{\varrho}$ we note that for $0<r<\frac{r_{0}}{2}$

$$
\frac{1}{r^{2}}[A(r(1+\delta))-A(r)]=\frac{A(r(1+\delta))}{r^{2}(1+\delta)^{2}}(1+\delta)^{2}-\frac{A(r)}{r^{2}} \rightarrow \mu\left(2 \delta+\delta^{2}\right)
$$

as $r \rightarrow 0$. Hence, given any $\varepsilon>0$, there exists an $r_{1}=r_{1}(\varepsilon, \delta)$, such that for $r<r_{1}$

$$
\frac{1}{r^{2}} \int_{r}^{r(1+\delta)} t \int_{k_{t}}\left|\varphi^{\prime}\left(t e^{i \theta}\right)\right|^{2} d \theta d t<\mu\left(2 \delta+\delta^{2}\right)+\varepsilon .
$$

Using $\varrho=r^{\prime}$ as determined in (2.12), we obtain by the same calculation 


$$
\int_{k_{\varrho}}\left|\varphi^{\prime}\left(\varrho e^{i \theta}\right)\right|^{2} d t<2 \mu+\frac{2 \varepsilon}{2 \delta+\delta^{2}} .
$$

Now, by (2.1) and (3.5)

$$
\frac{l_{\varrho}^{2}}{\varrho^{2}} \leqq \Theta(\varrho) \int_{k_{\varrho}}\left|\varphi^{\prime}\left(\varrho e^{i \theta}\right)\right|^{2} d \theta<2 \mu \Theta(\varrho)+\frac{2 \varepsilon \Theta(\varrho)}{2 \delta+\delta^{2}}
$$

and, therefore, for $\varrho=r^{\prime}$

$$
\varlimsup_{\varrho \rightarrow 0} \frac{l_{\varrho}^{2}}{\varrho^{2}} \leqq 2 \pi \mu+\frac{2 \varepsilon \pi}{2 \delta+\delta^{2}} .
$$

A completely analogous argument shows that for $\varrho=r^{\prime \prime}$ (Lemma 3)

$$
\varlimsup_{\varrho \rightarrow 0} \frac{l_{\varrho}^{2}}{\varrho^{2}} \leqq 2 \pi \mu+\frac{2 \varepsilon \pi}{2 \delta-\delta^{2}} .
$$

Thus we obtain for $\varrho=r^{\prime}$ and $\varrho=r^{\prime \prime}$, using the inequality on the left in $(3.3)$,

$$
\varlimsup_{\varrho \rightarrow 0}\left|\frac{\varphi(i \varrho)}{i \varrho}\right| \leqq\left\{\frac{2 \mu}{\pi}+\frac{2 \varepsilon}{\pi\left(2 \delta-\delta^{2}\right)}\right\}^{1 / 2} .
$$

Since this holds for every $\varepsilon>0$ we have for $\varrho=r^{\prime}$ and $\varrho=r^{\prime \prime}$

$$
\varlimsup_{\varrho \rightarrow 0}\left|\frac{\varphi(i \varrho)}{i \varrho}\right| \leqq \sqrt{\frac{2 \mu}{\pi}} .
$$

Combining (3.4), (3.7), and (3.1) we find for $z \in k_{o}$

$$
\lim _{z \rightarrow 0}\left|\frac{\varphi(z)}{z}\right|=\sqrt{\frac{2 \mu}{\pi}} .
$$

Finally, to show that (3.8) holds when $z \rightarrow z_{0}$ in the kernel $K$, we keep $\delta, \quad 0<\delta<1$, fixed and determine for a given $r$ the radii $r^{\prime}$ and $r^{\prime \prime}$. For any $z \in k_{r}$ there exist points $z^{\prime} \in k_{r^{\prime}}$ and $z^{\prime \prime} \in k_{r^{\prime \prime}}$ such that

$$
(1-\delta)\left|\frac{\varphi\left(z^{\prime \prime}\right)}{z^{\prime \prime}}\right|<\left|\frac{\varphi(z)}{z}\right|<\left|\frac{\varphi\left(z^{\prime}\right)}{z^{\prime}}\right|(1+\delta)
$$

(To find $z^{\prime}$ and $z^{\prime \prime}$ we merely determine proper points in which arg $\$=$ $\arg \varphi(z)$ intersects the $\operatorname{arcs} \varphi\left(k_{r^{\prime}}\right)$ and $\varphi\left(k_{r^{\prime \prime}}\right)$, respectively. The inverse images of these points are $z^{\prime}$ and $\left.z^{\prime \prime}\right)$. Now, as $r \rightarrow 0, z \in k_{r}$

$$
(1-\delta) \sqrt{\frac{2 \mu}{\pi}} \leqq \lim _{r \rightarrow 0}\left|\frac{\varphi(z)}{z}\right| \leqq \varlimsup_{r \rightarrow 0}\left|\frac{\varphi(z)}{z}\right| \leqq \sqrt{\frac{2 \mu}{\pi}}(1+\delta) .
$$


Since $\delta$ may be chosen arbitrarily small, (3.8) is valid as $z \rightarrow z_{0}$ in $K$. To complete the proof of our theorem we note that, because of hypothesis (C), for $z \in K, \lim _{z \rightarrow 0} \arg \frac{\varphi(z)}{z}=0$.

Remark. Under the hypotheses of Theorem 1 , for $z \in A_{\alpha}$ for any $x$, $0<\alpha<\frac{\pi}{2}, \lim _{z \rightarrow z_{0}} \varphi^{\prime}(z)=\varkappa . \quad($ see $(2.9))$.

\section{Existence of the derivative for unrestricted approach}

Suppose $C$ is a closed Jordan curve which passes through $z=0$. Let $z(t),-T<t \leqq T$ be a parametrization of $C$ such that $z(0)=0$. For $0<r<|z(T)|$ draw the circle $|z|=r$; let

$$
t_{r}=\max _{|z(t)|=r}|t|, \text { where }|t|<T,
$$

and

$$
\Delta(r)=\max _{|t| \leqq t_{r}}|z(t)|
$$

Thus the arc $z=z(t)$ for $|t| \leqq t_{r}$ is entirely contained in $z \leqq \Delta(r)$. We shall say that $C$ satisfies condition (U) at $z=0$ if

$$
\lim _{r \rightarrow 0} \frac{\Delta(r)}{r}=1^{4)} .
$$

This condition is independent of the parametrization of $C$.

Remark. Let $t_{r}^{*}=\min _{|z(t)|=r}|t|$ and $\delta(r)=\min _{t_{r}^{*} \leqq|t| \leqq t_{r}}|z(t)|$.

Then it is easily seen from the definition of $\Delta(r)$ that

$$
\Delta(\delta(r)) \geqq r .
$$

Theorem 2. Suppose that $C$ is a closed Jordan curve passing through $z=0$ which has the following properties:

(a) The $x$-axis is a tangent to $C$ at $z=0$ and the positive $y$-axis points in the direction of the interior normal.

(b) $C$ satisfies condition (U) at $z=0$.

(c) The interior domain $\Omega$ bounded by $C$ satisfies condition (B) of section 1.5)

4) In [17] this condition is called "reguläre Unbewalltheit».

${ }^{5}$ ) Conditions (A) and (C) are fulfilled since $C$ has a tangent at $z=0$. 
If $\zeta=\varphi(z)$ is the mapping function defined in section 1 , then

$$
\lim _{z \rightarrow 0} \frac{\varphi(z)}{z}=x(0 \leqq \varkappa<\infty
$$

exists for unrestricted approach in $\Omega \cup C$.

Proof. Let $\varepsilon$ be given, $0<\varepsilon<1$. Because of hypothesis (b) there exists an $R(\varepsilon)>0$ such that for $0<r \leqq R(\varepsilon)$

$$
\Delta(r)<(1+\varepsilon) r .
$$

Let $\delta(r)$ be defined as above (see Remark). Then $\Delta(\delta(r))<(1+\varepsilon) \delta(r)$, and since $J(\delta(r)) \geqq r$ we have

$$
r<(1+\varepsilon) \delta(r) .
$$

Thus we have the inequalities

$$
r(1-\varepsilon)<\frac{r}{1+\varepsilon}<\delta(r) \leqq r \leqq \Delta(r)<(1+\varepsilon) r .
$$

From the definitions of $\Delta(r)$ and $\delta(r)$ we note: if $z=r e^{i \theta} \in C$, then for any other point $z^{\prime} \in C$ with $\left|z^{\prime}\right|>\Delta(r)$ on the ssame side" of $z=0$ as $z$

$$
\left|\varphi\left(z^{\prime}\right)\right|>|\varphi(z)|
$$

and for any $z^{\prime \prime} \in C$ with $\left|z^{\prime \prime}\right|<\delta(r)$ on the "same side» of $z=0$ as $z$

$$
|\varphi(z)|>\left|\varphi\left(z^{\prime \prime}\right)\right| \text {. }
$$

Consider two circular cross cuts $k_{r^{\prime \prime}}$ and $k_{r^{\prime}}$ with $(1-\varepsilon) \delta(r) \leqq r^{\prime \prime}<\delta(r)$ and $\Delta(r)<r^{\prime} \leqq(1+\varepsilon) \Delta(r)$. Let $z^{\prime \prime}$ be an endpoint of $k_{r^{\prime \prime}}$ and $z^{\prime}$ and endpoint of $k_{r^{\prime}}\left(z^{\prime}\right.$ and $z^{\prime \prime}$ are points of $\left.C\right)$. Then for $z, z^{\prime}, z^{\prime \prime}$ on the "same side" of $z=0$

$$
\frac{r^{\prime \prime}}{r}\left|\frac{\varphi\left(z^{\prime \prime}\right)}{z^{\prime \prime}}\right| \leqq\left|\frac{\varphi(z)}{z}\right| \leqq\left|\frac{\varphi\left(z^{\prime}\right)}{z^{\prime}}\right| \frac{r^{\prime}}{r} .
$$

We let now $r \rightarrow 0$ and obtain from Theorem 1 and (4.1)

$$
\varkappa(1-\varepsilon)^{2} \leqq \lim _{r \rightarrow 0}\left|\frac{\varphi(z)}{z}\right| \leqq \varlimsup_{r \rightarrow 0}\left|\frac{\varphi(z)}{z}\right|=\varkappa(1+\varepsilon)^{2} .
$$

Since $\varepsilon$ is arbitrary, we have for approach along $C$ from either side of $z=0$,

$$
\lim _{z \rightarrow 0}\left|\frac{\varphi(z)}{z}\right|=\varkappa .
$$

By a well known theorem of Lindelöf 


$$
\lim _{z \rightarrow 0} \arg (\varphi(z) / z)=0, \quad(z \in \Omega \cup C)
$$

so that we have as $z \rightarrow 0$ along $C$

$$
\lim _{z \rightarrow 0} \frac{\varphi(z)}{z}=\varkappa(\geqq 0) \text {. }
$$

To prove that (4.2) holds also for unrestricted approach in $\Omega \cup C$ it is sufficient to show that $\left|\frac{\varphi(z)}{z}\right|$ is bounded in $\Omega$. Let $\left\{k_{\varrho_{n}}\right\}$ be a sequence of circular cross cuts with $\lim _{n \rightarrow \infty} \varrho_{n}=0$. Then $\Omega$ may be exhausted by a sequence of Jordan domains $\left\{\Omega_{n}\right\}$ bounded by $k_{\varrho_{n}}$ and an arc $\gamma_{n}$ of $C$ with does not contain $z=0$. By Lemma $1,\left|\frac{\varphi(z)}{z}\right| \leqq M$ on all $k_{\varrho_{n}}$ and by (4.2), $\left|\frac{\varphi(z)}{z}\right| \leqq M^{\prime}$, for a suitable $M^{\prime}$ on $C(z \neq 0)$. Since $\left|\frac{\varphi(z)}{z}\right|$ is continuous in the closure of each $\Omega_{n}$ it follows that it is uniformly bounded in all $\Omega_{n}$ and hence in $\Omega$. This completes the proof.

\section{Conditions for $x$ to be positive}

To show that $x>0$ it is sufficient to find a domain $\Omega_{1} \subset \Omega$ which has $z_{0}=0$ as an accessible boundary point along the $y$-axis, such that for its mapping function $\varphi_{1}(z)$ onto $\operatorname{Im}(\zeta)>0$, with $\varphi_{1}\left(z_{0}\right)=0$, $\frac{\lim }{y_{\curlyvee} 0}\left|\frac{\varphi_{1}(i y)}{y}\right|>0$. In particular, any $\Omega_{1} \subset \Omega$ for which $\varphi_{1}$ has a nonvanishing angular derivative may be used. We give the following simple and quite general criterion. $\Omega, \varphi$ are defined in $\S 1, x=\lim _{y \rightarrow 0} \frac{|\varphi(i y)|}{y}$.

Theorem 3. Suppose that, for all real $x, h(x)$ is a non-negative, continuous, even function, such that

$$
\text { (a) } \quad \int_{0}^{\delta} \frac{h(x)}{x^{2}} d x<\infty(\delta>0) \text { and (b) } \int_{x-h(x)}^{x+h(x)} h(t) d t \geqq c h^{2}(x)
$$

for some constant $c>0$. If for some $a>0, y_{0}>0$ the domain $\left\{z=x+i y \mid-a<x<a, h(x)<y<y_{0}\right\} \subset \Omega$, then $\left.x>0 .{ }^{6}\right)$

$\left.{ }^{6}\right)$ Our Theorem 3 combined with Theorem 1 is sharper than the earlier criteria for existence of the nonvanishing angular derivative cited in [9] pp. 22-28 and some of the more recent ones in [6], Chapter VI. However it is restricted by the fact that the curve $y=h(x)$ and hence the domain $\Omega_{1}$ which we construct are contained in $y \geqq 0$. For the case that $\Omega$ is an infinite domain with $z_{0}$ at $\infty$ and is mapped onto a parallel strip we proved a similar result in [19], Theorem 6; cf. also the Remark in [19] p. 100 where comparisons with earlier criteria are given. 
Remarks 1. The continuity of $h$ combined with (a) implies $h(0)=0$, and (b) combined with (a) imply $h(x)=o(x)$ as $x \rightarrow 0$.

2. Condition (b) is clearly satisfied if $h(x)$ is monotonically increasing for $x>0$; in that case the theorem yields a well known result. Other sufficient conditions that (b) hold are that, for some constant $k>0$. either

$$
\frac{h(t)-h(x)}{t-x} \geqq-k \text { or } \frac{h(t)-h(x)}{t-x} \leqq k(t>x) .
$$

These inequalities facilitate the application of our theorem in many cases.

Proof. $\left.{ }^{7}\right)$ We may assume $2 a<y_{0}$. Traverse the circle $\mid z-i a=a$ from the point $z=2 a i$ in both directions to the first points of intersection, $\pm b+i h(b)$, with the curve $y=h(x)$ and denote the (circular) aro so described by $\Gamma$. Clearly $\Gamma \subset \Omega$. Then $\Gamma$ and the arc $\gamma=\{x \leqq b$, $y=h(x)\}$ form a closed Jordan curve contained in $\bar{\Omega}$. Denote its interior by $\Omega_{1} ; \Omega_{1} \subset \Omega$. We may assume $b>0$, for $b=0$ implies that $\Omega_{1}$ is the disk $|z-i a|=a$, and in this case the conclusion of our theorem is well known. We may also suppose $h(x) \leqq \frac{1}{2}|x| \leqq \frac{1}{2} a$ for $\mid x \leqq b$ (by taking a sufficiently small).

Let $G_{1}(z, i a)=\log \frac{a}{r}-v(z)$ denote the Green's function of $\Omega_{1}$ with the pole at $z=i a$, where $r=|z-i a|$ and $v(z)$ is harmonic in $\Omega_{1}$. For $z \in \Gamma \cup \gamma$ we have $v(z)=\log \frac{a}{r}$.

Now for $z=x+i y \in \gamma$ we have, if $r<a$,

$$
\log \frac{a}{r}=\log \left(1+\frac{a-r}{r}\right)<\frac{a-r}{r} \leqq \frac{2}{a}(a-r) \leqq \frac{2}{a} h(x)
$$

and the inequality between the first and last term still holds if $r \geqq a$, since then $\log \frac{a}{r} \leqq 0$. Thus for $z \in \gamma$

$$
v(z) \leqq \frac{2}{a} h(x) \quad(z=x+i h(x)) .
$$

Let

$$
u(z)=\frac{1}{\pi} \int_{-2 a}^{2 a} h(t) \frac{y d t}{(t-x)^{2}+y^{2}}
$$

7) The proof generalizes a method of M. Tsuji [12] p. 368. To make this paper self-contained we include the proof, although it is similar to that of our Theorem 6 of [19]. 
which is harmonic in $y>0$. For $z \in \gamma \quad$ (if $y=0, u(z)=h(x)=0)$ :

$$
u(z) \geqq \frac{1}{\pi} \int_{x-y}^{x+y} h(t) \frac{y d t}{(t-x)^{2}+y^{2}} \geqq \frac{1}{2 \pi y} \int_{x-y}^{x+y} h(t) d t \geqq \frac{c h(x)}{2 \pi}
$$

since $|t-x| \leqq y$ and by property (b). Hence, by (5.1) for $z \in \gamma$

$$
v(z) \leqq \frac{4 \pi}{a c} u(z)
$$

Since on $\Gamma, v(z)=0$ and $u(z)>0$ for $y>0$, (5.2) holds in $\Omega_{1}$. Now, for $z=i y, \quad 0<y<a$,

$$
u(i y)=\frac{1}{\pi} \int_{-2 a}^{2 a} h(t) \frac{y d t}{t^{2}+y^{2}} \leqq \frac{y}{\pi} \int_{-2 a}^{2 a} \frac{h(t)}{t^{2}} d t
$$

and thus

$$
v(i y) \leqq \frac{4 y}{a c} \int_{-2 a}^{2 a} \frac{h(t)}{t^{2}} d t
$$

Hence we have for $0<y<\frac{a}{2}(a-r=y)$, by $(5.3)$,

$$
\begin{gathered}
G_{1}(i y, i a)=\log \left(1+\frac{a-r}{r}\right)-v(i y) \geqq \frac{1}{2} \frac{a-r}{a}-v(i y)=\frac{y}{2 a}-v(i y) \\
\geqq \frac{y}{a}\left\{\frac{1}{2}-\frac{4}{c} \int_{-2 a}^{2 a} \frac{h(t)}{t^{2}} d t\right\}=y A .
\end{gathered}
$$

We can choose a priori $a$ so small that $A>0$. If $G(z, i a)$ denotes the Green's function of $\Omega$ with pole at $z=a i$, we have for $z \in \Omega_{1}$, $G(z, i a) \geqq G_{1}(z, i a)$ and consequently

$$
\frac{\lim }{y \downarrow 0} \frac{G(i y, i a)}{y} \geqq \frac{\lim }{y \downarrow 0} \frac{G_{1}(i y, i a)}{y} \geqq A>0 .
$$

Suitable linear transformations applied to the mapping function $\varphi$ show then that $x>0$. 


\section{APPENDIX}

We indicate a direct proof of (1.1) stated in section 1.

Theorem. If the domain $\Omega$ satisfies conditions (A) and (C) of section 1 , then $\lim _{z-z_{0}} \arg \frac{\varphi(z)}{z}=0$, uniformly as $z \rightarrow z_{0}$ in $K$.

The function $w=\log \frac{i}{z}, w=u+i v$, maps $\Omega$ onto a simply connected domain $S$ which contains a part $L:\left\{u \geqq u_{0}, v=0\right\}$ of the real axis, for some $u_{0}$. Let $w_{\infty}$ denote the boundary point at $w=\infty$ of $S$ accessible along $L$ (corresponding to $z_{0}$ ). For $u \geqq u_{0}$ let $\theta_{u}$ be the largest (open) segment in $S$ which intersects $L$ (corresponding to $k_{r}$ ) and let $\Theta(u)$ be its length. The domain $\Gamma=\left\{w \in \theta_{u}, u \geqq u_{0}\right\}$ is the »kernel» of $S$ at $w_{\infty}$ (depending on $u_{0}$ ). Similarly $Z=\log \frac{i}{\zeta}, Z=X+i Y$, maps the halfplane $\operatorname{Im}(\zeta)>0$ onto the infinite parallel strip $\Sigma=\left\{Y<\frac{\pi}{2}\right.$, $-\infty<X<\infty\}$ and the function $Z(w)=X(w)+i Y(w)=$ $-\log \left[i^{-1} \varphi\left(i e^{-w}\right)\right]$ maps $S$ conformally onto $\Sigma$ such that $\lim X(u)=-\infty$. For all sufficiently large $u, Z(w)$ maps $\theta_{u}$ onto an $\begin{gathered}u \rightarrow+\infty \\ \operatorname{arc} \sigma_{u} \subset \Sigma\end{gathered}$ connects a (finite) point of $Y=\frac{\pi}{2}$ to a (finite) point on $Y=-\frac{\pi}{2}$. We assume that $u_{0}$ is so large that this is the case for $u \geqq u_{0}$. The cross cut $\theta_{u_{0}}$ divides $S$ into two subdomains; let $S_{0}$ denote the one containing the part of $L$ with $u>u_{0}$.

Let $\alpha$ be an endpoint of a $\theta_{u}$ for $u>u_{0}$. We describe a circle $C^{\prime}$ "of radius $\varrho$ about $\alpha$ where $\varrho<\operatorname{Min}\left(u-u_{0},|\alpha-u|\right)$ so that $C_{\varrho}$ does not intersect $\theta_{u_{0}}$ and the $u$-axis. $C_{\varrho}$ crosses $\theta_{u}$ at a point $\alpha^{\prime}$. Let $k_{\text {, denote the }}$ largest (open) arc of $C_{o}$ which contains $\alpha^{\prime}$ and is contained in $s$ : it is also contained in $S_{0} ; k_{o}$ divides $S_{0}$ into two domains and the one containing the segment $\alpha \alpha^{\prime}$ of $\theta_{u}$ will be denoted by $D_{0}$. Then we have (Wolff [22], Warschawski [19] p. 84):

Lemma. For every $\delta, 0<\delta<\operatorname{Min}\left(e^{-32}, u-u_{0},|\alpha-u|\right)$ there exists $a \varrho, \quad \delta^{2}<\varrho<\delta$, such that the image of $D_{\varrho}$ under the mapping $w \rightarrow Z(w)$ $i s$ a domain $\Delta_{\varrho}$ bounded by the image of $k_{\varrho}$ and a finite segment of the line $Y=\frac{\pi}{2}$ or $Y=-\frac{\pi}{2}$. The diameter of $\Delta_{\varrho}$ does not exceed $M\left(\log \frac{1}{\delta}\right)^{-1 / 2}$ where $M \leqq 6 \pi \sqrt{2}$.

In particular, we have for $w_{1}, w_{2} \in D_{0}$,

$$
\left|Y\left(w_{1}\right)-Y\left(w_{2}\right)\right| \leqq M\left(\log \frac{1}{\delta}\right)^{-1 / 2},
$$


and if we let $w_{1} \rightarrow \alpha$ along $\theta_{u}$, for any $w \in D_{\varrho}$,

$$
\left|\frac{\pi}{2}-\right| Y(w)|| \leqq M\left(\log \frac{1}{\delta}\right)^{-1 / 2} .
$$

Now, to prove the theorem, assume $u>u_{0}$ and choose any $\varepsilon>0$ such that $\sqrt{\bar{\varepsilon}}<\operatorname{Min}\left(e^{-32}, u-u_{0},|\alpha-u|\right)$ where $\alpha$ (as above) is an endpoint of $\theta_{u}$. Then there exists an $R_{\varepsilon}$ such that (because of (A))

$$
S_{\frac{\pi}{2}-\varepsilon}=\left\{w=u+i v\left|u \geqq R_{\varepsilon},\right| v \mid \leqq \frac{\pi}{2}-\varepsilon\right\} \subset S,
$$

and (because of $(\mathrm{C}))$ for $u>R_{\varepsilon}, \quad w=u+i v \in \theta_{u}$

(ii) for $v>0$ : $v<\frac{\pi}{2}+\varepsilon$ and for $v<0$ : $v>-\frac{\pi}{2}-\varepsilon$.

Applying (A.l) with $\delta^{2}=\varepsilon$ we see that for every $w \in \theta_{u}, u>R_{s}$, and $|v| \geqq \frac{\pi}{2}-\varepsilon$

$$
\left|\frac{\pi}{2}-\right| Y(w)|| \leqq 2 M\left(\log \frac{1}{\varepsilon}\right)^{-1 / 2}
$$

Hence, for every such $w$ with $v \geqq \frac{\pi}{2}-\varepsilon$, by (ii)

$$
|Y(w)-v| \leqq Y(w)-\frac{\pi}{2}+\left|v-\frac{\pi}{2}\right| \leqq 2 M\left(\log \frac{1}{\varepsilon}\right)^{-12}+\varepsilon
$$

and for $w$ with $v \leqq-\frac{\pi}{2}+\varepsilon$

$$
|Y(w)-v| \leqq\left|\frac{\pi}{2}+Y(w)\right|+\mid \frac{\pi}{2}+v \leqq 2 M\left(\log \frac{1}{\varepsilon}\right)^{-12}+\varepsilon
$$

Finally since $Y(w)-v$ is harmonic and bounded in $S_{\frac{\pi}{2}-\varepsilon}$ and (A.2) and (A.3) are satisfied on the horizontal boundaries of this strip, it follows that for $w \in S_{\frac{\pi}{2}-\varepsilon}$, uniformly,

$$
\left.\varlimsup_{u \rightarrow+\infty} \mid Y(w)-v\right) \mid \leqq 2 M\left(\log \frac{1}{\varepsilon}\right)^{-1 / 2}+\varepsilon
$$


A. I. 420

But by (A.2) and (A.3) it is also true uniformly for $w \in \Gamma$ outside of $S_{\frac{\pi}{2}-\varepsilon}$ as $u \rightarrow+\infty$. Hence $\lim _{u \rightarrow+\infty}(Y(w)-v)=0$, uniformly for $w \in \Gamma$. This is equivalent to the conclusion of the theorem.

University of California, San Diego

La Jolla, California 


\section{Bibliography}

1. L. Ahlfors, Untersuchungen zur Theorie der konformen Abbildung und der ganzen Funktionen, Acta Societatis Scientiarum Fennicæ (n.s.) A, 1 (1930): $1-40$.

2. C. Carathéodory, Über die Winkelderivierten von beschränkten analytischen Funktionen, Sitz. Berichte, Berliner Akad. der Wissenschaften, 32 (1929): $39-54$.

3. E. T. Copson, Introduction to the theory of functions of a complex variable, Oxford Univ. Press, 1935.

4. J. Dufresnoy et J. Ferrand, Extension d'une inégalité de M. Ahlfor's et application au problème de la dérivee angulaire, Bulletin des Sciences Mathématiques 69 (1945): $165-174$.

5. J. Ferrand, Sur l'inégalité d'Ahlfors et son application au problème de la dérivée angulaire, Bulletin de la Société Math. de France, 72 (1944): 178-192.

6. J. Lelong-Ferrand, Représentation conforme et transformations a intégrale de Dirichlet bornée, Gauthier-Villars, Paris, 1955.

7. C. Gattegno, Nouvelle démonstration d'un théorème de M. Ostrowski sur la représentation conforme, Bulletin des Sciences Math. 62 (1938) $12-21$.

8. C. Gattegno et A. Ostrowski, Représentation conforme à la frontière: domains generaux, Mémorial des Sciences Math. Fasc. 109 (1949), Gauthier-Villars.

9. - - Représentation conforme à la frontière: domains particuliers, ibid. Fase. $110(1949)$.

10. A. Ostrowskr, Über den Habitus der konformen Abbildung on Rande des Abbildungsbereiches, Acta Math. 64 (1934): $81-185$.

11. - - Zur Randverzerrung bei konformer Abbildung. Prace Matematiczno Fiziczne, 44 (1937): $371-471$.

12. M. Tsuj, Potential theory in modern function theory, Maruzen Co., Ltd., Tokyo, 1959.

13. J. G. VAN Der Corput, Über die Winkelableitung bei konformer Abbildung, Proc. Ned. Akademie van Wetenschappen, 35 (1932): 330-338.

14. C. VIssER, Über beschränkte analytische Funktionen und die Randverhältnisse bei konformen Abbildungen, Nath. Annalen 107 (1932): 28-39.

15. …- Über die Ränderzuordnung bei konformen Abbildungen, Proc. Ned. Akad. van Wetenschappen 38 (1935): $411-414$.

16. -....-. Sur la dérivée angulaire des fonctions univalentes: I. Proc. Ned. Akad. van Wetenschappen 38 (1935); $402-41$; II. 40 (1937): $223-226$.

17. S. E. WarschawsKi, Über das Randverhalten der Ableitung der Abbildungsfunktion bei konformer Abbildung, Math. Zeitschrift 35 (1932): $322-456$.

18. - - Zur Randverzerrung bei konformer Abbildung, Compositio Mathematica 1 (1.935): $314-343$.

19. "- On the boundary behavior of conformal maps, Nagoya Mathematical Journal 30 (1967): $83-101$. 
20. J. WolfF, Sur une géneralization d'un lemme de Schwarz, C. R. Acad. Sc. Paris 183 (1926): $500-502$.

21. - - Sur la représentation conforme des bandes, Compositio Mathematica 1 (1934): $217-222$.

22. - - Démonstration d'un théorème sur la conservation des angles dans la représentation conforme on voisinage d'un point frontière, Proc. Ned. Akad. van Wetenschappen, 38 (1935): $46-50$. 